\title{
A COMPARISON THEOREM FOR HAMILTONIAN VECTOR FIELDS
}

\author{
ALAN WEINSTEIN ${ }^{1}$ AND JERROLD MARSDEN ${ }^{2}$
}

\begin{abstract}
The question of completeness of Hamiltonian systems is investigated for a class of potentials not necessarily bounded below. The result generalizes previous work of W. Gordon and D. Ebin.
\end{abstract}

This paper extends the completeness theorem of Ebin [1] to include certain potential functions $V$ not necessarily bounded below. The condition on $V$ is essentially the same as a condition for a corresponding quantum mechanical theorem. See [3] and Remark 3 below. We shall prove a comparison theorem which reduces the general case to the one-dimensional case, so we begin with the latter.

1. The one-dimensional case. Let $R^{+}$be the nonnegative reals and $V_{0}: R^{+} \rightarrow R$ a nonincreasing $C^{1}$ function. Consider the Hamiltonian system with the usual kinetic energy and potential $V_{0}$; i.e. if $c(l)$ is a solution curve we have

$$
c^{\prime \prime}(t)=-\frac{d V_{0}}{d x}(c(t)) .
$$

By monotonicity of $V_{0}$, if $c^{\prime}(0) \geqq 0$ then $c^{\prime}(t) \geqq 0$ for all $t \geqq 0$. Thus if $H=\left[c^{\prime}(t) / 2\right]^{2}+V_{0}(c(t))$ is the constant total energy,

$$
c^{\prime}(t)=2\left(H-V_{0}(c(t))\right)^{1 / 2} .
$$

Definition. The potential $V_{0}$ is positively complete iff

$$
\int_{x_{1}}^{x} \frac{d x}{\left(2\left(H-V_{0}(x)\right)\right)^{1 / 2}} \rightarrow \infty \quad \text { as } x \rightarrow \infty
$$

for all $x_{1} \geqq 0$ and $H$ such that $V_{0}\left(x_{1}\right)<H$.

It is easy to see that if this holds for some $x_{1}, H$, such that $V_{0}\left(x_{1}\right)<H$ then it holds for all such $x_{1}, H$ (use the fact that improper integrals with asymptotic integrands are simultaneously convergent or divergent).

Received by the editors March 15, 1970.

AMS 1969 subject classifications. Primary 3465; Secondary 3442.

Key words and phrases. Complete vector field, infinite-dimensional manifold, Hamiltonian vector field, dissipative system.

1 Partially supported by NSF Grant GP-13348.

${ }^{2}$ Partially supported by Navy contract N00014-69-A-0200-1002. 
Since the above integral is just the time required for $c(t)$ to move from $x_{1}$ to $x$ we see that $V_{0}$ is positively complete iff all integral curves $c(t)$ with $c(0) \geqq 0, c^{\prime}(0) \geqq 0$ are defined for all $t \geqq 0$. (The case when $c^{\prime}(0)=0$ is easily disposed of.)

Below we will use the notation $\tilde{c}\left(x_{0}, H\right)(t)$ for the integral curve with $\tilde{c}\left(x_{0}, H\right)(0)=x_{0}$ and energy $H$.

EXAmPle. The function $-x^{\alpha}$ for $\alpha \geqq 0$ is positively complete iff $\alpha \leqq 2$. The same is true for

$$
-x[\log (x+1)]^{\alpha}, \quad-x \log (x+1)[\log (\log (x+1)+1)]^{\alpha} \text { etc. }
$$

\section{The general case.}

Theorem. Let $M$ be a complete Riemannian manifold (possibly infinite-dimensional) and let $V$ be a $C^{1}$ function on $M$. Suppose there is a point $p \in M$ and a positively complete $V_{0}$ on $R^{+}$such that for all $m \in M_{1}$ [with $d(m, p)$ sufficiently large], $V(m) \geqq V_{0}(d(m, p))$ where $d$ is the Riemannian distance on $M$. Then the flow on $T M$ of the Hamiltonian vector field with (the usual kinetic energy $K(v)=\langle v, v\rangle / 2$ and) potential $V$ is a complete flow (i.e. integral curves are defined for all $t \in R$ ).

ExAmples. If $V(m) \geqq-($ Constant $) d(m, p)^{2}$ for sufficiently large $d(m, p)$ the conditions hold. This is satisfied if $\|\operatorname{grad} V(m)\|$ $\leqq($ Constant $) d(m, p)$ (for sufficiently large $d(m, p)$ ).

Proof of Theorem. Let $c:[0, b) \rightarrow T M$ be an integral curve, $0<b$ $<\infty$. By the arguments in [1], it will suffice to show that the curve $c_{0}(t)$, the projection of $c(t)$ on $M$, remains in a bounded set for all $t \in[0, b)$ (a similar argument holds for $t \in(-b, 0])$.

Let $n=c_{0}(0)$ and $H$ the energy of $c(t)$. Let

$$
f_{1}(t)=d\left(c_{0}(t), p\right) \text { and } f_{2}(t)=\tilde{c}(d(n, p), H)(t)
$$

(notation as in $\$ 1$ ). Now

$$
\begin{aligned}
f_{1}(t) & \leqq d(p, n)+\int_{0}^{t}\|c(s)\| d s \\
& =d(p, n)+\int_{0}^{t}\left(2\left[H-V\left(c_{0}(s)\right)\right]\right)^{1 / 2} d s \\
& \leqq d(p, n)+\int_{0}^{t}\left(2\left[H-V_{0}\left(f_{1}(s)\right)\right]\right)^{1 / 2} d s .
\end{aligned}
$$

Also we have

$$
f_{2}(t)=d(p, n)+\int_{0}^{t}\left(2\left[H-V_{0}\left(f_{2}(s)\right)\right]\right)^{1 / 2} d s .
$$


It follows from these and monotonicity of $V_{0}$ that

$$
f_{1}(t) \leqq f_{2}(t) \leqq \tilde{c}(d(n, p), H)(b)
$$

for all $t \in[0, b)$. (See $[2$, p. 29] for the proof of a similar result.) We conclude that $f_{1}(t)=d\left(c_{n}(t), p\right)$ remains bounded for $t \in[0, b)$ and so the result follows.

REMARKs. (1) The completeness for $t \geqq 0$ is preserved if a dissipative vector field $Y$ is added to the Hamiltonian vector field (i.e. $Y$ is vertical $[T \pi(Y)=0]$ and $Y \cdot K \leqq 0$ where $K$ is the kinetic energy). This is easy to see.

(2) This proof also gives an estimate for the growth of $d\left(c_{0}(t), p\right)$ in terms of $V_{0}$; for example if $V_{0}=-x^{2}$ then $d\left(c_{0}(t), p\right)-d(n, p)$ grows like $e^{t}$.

(3) In [3] (and earlier papers) it is shown that on $R^{n}$ for potentials $V$ satisfying essentially the condition here (of bounded below by a positively complete function), the corresponding quantum mechanical flows are "complete" i.e. the operator is essentially selfadjoint. In fact the result may be generalized to arbitrary complete Riemannian manifolds. (See P. Chernoff and J. Marsden, Hamiltonian systems and quantum mechanics (in preparation).)

(4) See [4] for some related results.

\section{REFERENCES}

1. D. Ebin, Completeness of Hamiltonian vector fields, (preprint).

2. P. Hartman, Ordinary differential equations, Wiley, New York, 1964. MR 30 $\# 1270$.

3. T. Ikebe and T. Kato, Uniqueness of the self-adjoint extension of singular elliptic differential operators, Arch. Rational Mech. Anal. 9 (1962), 77-92. MR 26 \#461.

4. J. Lelong-Ferrand, Sur les groupes à un paramétre de transformations des variétés différentiables, J. Math. Pures Appl. (9) 37 (1958), 269-278. MR 20 \#4874. , Condition pour qu'un groupe de transformations infinitésimales engendre un groupe global, C. R. Acad. Sci. Paris 249 (1959), 1852-1854. MR 22 \#218.

University of California, Berkeley, California 94720 